\title{
Photophysical studies of zinc phthalocyanine and chloroaluminum phthalocyanine incorporated into liposomes in the presence of additives
}

S.M.T. Nunes,

F.S. Sguilla and

A.C. Tedesco

\author{
Departamento de Química, Faculdade de Filosofia, \\ Ciências e Letras de Ribeirão Preto, Universidade de São Paulo, \\ Ribeirão Preto, SP, Brasil
}

\section{Correspondence \\ A.C. Tedesco \\ Departamento de Química \\ FFCLRP, USP \\ Av. Bandeirantes, 3900 \\ 14040-901 Ribeirão Preto, SP \\ Brasil \\ Fax: +55-16-633-8151 \\ E-mail: tedesco@ffclrp.usp.br \\ Research supported by FAPESP.}

Received January 9, 2003 Accepted September 9, 2003

\section{Abstract}

The photophysical properties of zinc phthalocyanine (ZnPC) and chloroaluminum phthalocyanine (AlPHCl) incorporated into liposomes of dimyristoyl phosphatidylcholine in the presence and absence of additives such as cholesterol or cardiolipin were studied by timeresolved fluorescence, laser flash photolysis and steady-state techniques. The absorbance of the drugs changed linearly with drug concentration, at least up to $5.0 \mu \mathrm{M}$ in homogeneous and heterogeneous media, indicating that aggregation did not occur in these media within this concentration range. The incorporation of the drugs into liposomes increases the dimerization constant by one order of magnitude (for $\mathrm{ZnPC}, 3.6 \times 10^{4}$ to $1.0 \times 10^{5} \mathrm{M}^{-1}$ and for $\mathrm{AlPHCl}$, $3.7 \times 10^{4}$ to $\left.1.5 \times 10^{5} \mathrm{M}^{-1}\right)$, but this feature dose does not rule out the use of this carrier, since the incorporation of these hydrophobic drugs into liposomes permits their systemic administration. Probe location in biological membranes and predominant positions of the phthalocyanines in liposomes were inferred on the basis of their fluorescence and triplet state properties. Both phthalocyanines are preferentially distributed in the internal regions of the liposome bilayer. The additives affect the distribution of these drugs within the liposomes, a fact that controls their delivery when both are used in a biological medium, retarding their release. The addition of the additives to the liposomes increases the internalization of phthalocyanines. The interaction of the drugs with a plasma protein, bovine serum albumin, was examined quantitatively by the fluorescence technique. The results show that when the drugs were incorporated into small unilamellar liposomes, the association with albumin was enhanced when compared with organic media, a fact that should increase the selectivity of tumor targeting by these phthalocyanines (for ZnPC, $0.71 \times 10^{6}$ to $1.30 \times 10^{7}$ $\mathrm{M}^{-1}$ and for AlPHCl, $4.86 \times 10^{7}$ to $\left.3.10 \times 10^{8} \mathrm{M}^{-1}\right)$.
Key words

- Zinc phthalocyanine

- Chloroaluminum

phthalocyanine

- Bovine serum albumin

- Liposomes

- Cholesterol

- Cardiolipin

- Dimyristoyl

phosphatidylcholine 


\section{Introduction}

Photodynamic therapy (PDT) of cancer is a noninvasive treatment of small and superficial tumors that is currently being used in a number of countries (1). The therapy is based on the systemic administration of a tumor-localizing photosensitizer followed by illumination with light of appropriate wavelength. The resulting photodynamic reactions give rise to singlet oxygen $\left({ }^{1} \mathrm{O}_{2}\right)$ and to other active oxygen species that lead to tumor destruction (1). The efficiency of PDT depends on the development of new drugs and the ability of these drugs to accumulate selectively in tumor tissues in comparison with normal tissues. Several new classes of photosensitizers for PDT have reached the stage of clinical trials during the past few years (2). Among the more promising second-generation photosensitizers are phthalocyanines (Figure 1). There has been considerable interest in phthalocyanines for use in PDT, mainly because of their high absorbance coefficient (at 650-680 nm), with optimal tissue penetration by light (3). The photophysical properties of phthalocyanines are strongly dependent on the central metal ion. Among the metal phthalocyanines, $\mathrm{Zn}$ (II) and $\mathrm{Al}$ (III) complexes (zinc phthalocyanine, $\mathrm{ZnPC}$, and chloroaluminum phthalocyanine, AlPHCl) present the most favorable photophysical properties for applica-

Figure 1. Typical phthalocyanine structure where $M$ is the central metal ion (Al, Zn, Co, Ga, Si) and $R$ represents a multitude of possible ring substituents including $\mathrm{SO}_{3} \mathrm{H}, \mathrm{F}, \mathrm{COOH}$, etc.

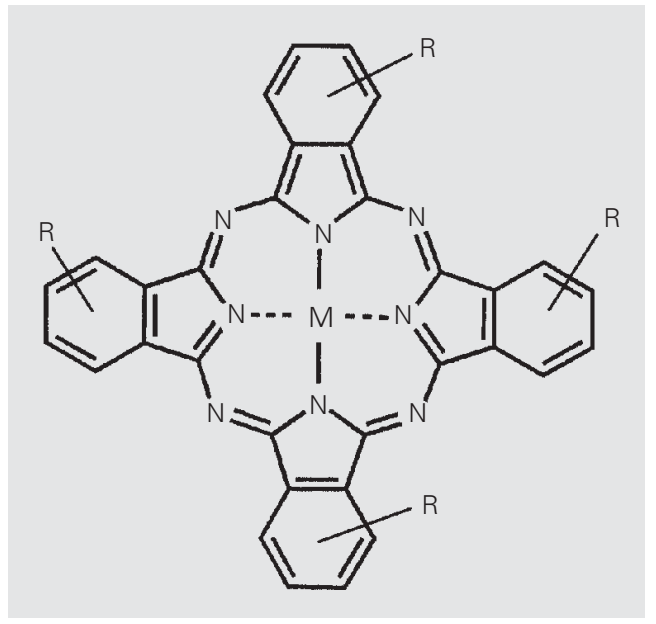

tion in PDT (4), i.e., relatively long-lived excited singlet states (ca. 3-8 ns) and longlived triplet states that are produced in high quantum yields (5).

Unfortunately, these phthalocyanines ( $\mathrm{ZnPC}$ and $\mathrm{AlPHCl})$ are insoluble in water or biologically compatible solvents. Thus, they must be administered in vivo by means of delivery systems (6). The transport of porphyrins in the bloodstream via liposomes has been shown to provide a larger and more selective accumulation of the drugs in neoplastic tissues (7), a fact that is a basic requirement for PDT. Their association with serum proteins can also enhance the preferential uptake of hydrophobic photosensitizers by tumor tissues, since serum albumin is one of the key components in blood that influences drug distribution. Since the intrinsic fluorescence of proteins is usually quenched upon the binding of tetrapyrrolic compounds (8), this spectroscopic behavior provides a means to study the interaction between these drugs and bovine serum albumin (BSA), permitting the determination of the binding constant, $K_{\mathrm{b}}$, and of the binding stoichiometry of the complex formed (9).

Nevertheless, phthalocyanines are prone to self-aggregation. The deviation from linear Beer-Lambert behavior for these drugs in solution caused by dimer formation is normally more pronounced in water than in organic solvents. Dimers are reported to be inactive or much more inefficient than monomers as photosensitizers (10). Unfortunately, phthalocyanines have been reported to display a strong tendency to form dimers in water as a result of the large hydrophobic skeleton avoiding contact with the aqueous medium (11), leading to the dimerization process and affecting their photophysical and photosensitizing properties and photodynamic action (12). Aggregation was found to reduce the sensitizing ability of phthalocyanines in electron transfer reactions and in $\mathrm{O}_{2}\left({ }^{1} \Delta_{\mathrm{g}}\right)$ photoproduction (13). Since incorporation of photosensitizers into liposomes 
(used as a drug delivery system) results in a high local concentration, information about the behavior of these drugs in these media is important for the understanding of their action in biological media.

In the present investigation we studied the aggregation parameters of $\mathrm{ZnPC}$ and $\mathrm{AlPHCl}$ in order to accurately determine the photophysical properties of these drugs. The predominant positions of these phthalocyanines in dimyristoyl phosphatidylcholine (DMPC) liposomes are analyzed, as well as the triplet state lifetime and the binding of these exogenous drugs with BSA.

\section{Material and Methods}

\section{Reagents}

$\mathrm{ZnPC}$, AlPHCl, methylviologen $\left(\mathrm{MV}^{2+}\right)$, 9,10-anthraquinone-2-sulfonate ( $\mathrm{AQS}^{-}$, sodium salt), and 9,10-anthraquinone-2,6disulfonate (AQDS ${ }^{2-}$, disodium salt) were purchased from Aldrich Chemical Company Inc. (Milwaukee, WI, USA) and used without further purification. DMPC, cardiolipin, cholesterol, and phosphate-buffered saline (PBS) were obtained from Sigma (St. Louis, MO, USA). All other chemicals were commercially available reagents of at least analytical grade.

All experiments were carried out with a phthalocyanine concentration of $5.0 \mu \mathrm{M}$. Stock solutions of $\mathrm{ZnPC}$ and AlPHCl (1.0 $\mathrm{mM}$ ) were routinely prepared in ethanol and DMSO, respectively, and stored in the dark at $4^{\circ} \mathrm{C}(\mathrm{ZnPC}$ was dissolved in $0.1 \%$ pyridine in ethanol, $v / v)$. The concentrations of the phthalocyanines were estimated spectrophotometrically using $\varepsilon_{678}=2.93 \times 10^{5} \mathrm{M}^{-1} \mathrm{~cm}^{-1}$ for AlPHCl in DMSO and liposomes and $\varepsilon_{673}=2.41 \times 10^{5} \mathrm{M}^{-1} \mathrm{~cm}^{-1}$ for $\mathrm{ZnPC}$ in ethanol and liposomes. Stock solutions of $\mathrm{MV}^{2+}\left(0.3 \mathrm{M}, \varepsilon=20,500 \mathrm{M}^{-1} \mathrm{~cm}^{-1}\right.$ at $\left.257 \mathrm{~nm}\right)$ and AQDS ${ }^{2-}\left(17 \mathrm{mM}, \varepsilon=6760 \mathrm{M}^{-1} \mathrm{~cm}^{-1}\right.$ at $328 \mathrm{~nm}$ ) in water were stored in the dark at $-15^{\circ} \mathrm{C}$. Stock solutions of $\mathrm{AQS}^{-}(17 \mathrm{mM}$, $\varepsilon=5450 \mathrm{M}^{-1} \mathrm{~cm}^{-1}$ at $330 \mathrm{~nm}$ ) in water were stored in the dark at room temperature. The values of the extinction coefficients were obtained from the literature (14).

\section{Liposome preparation}

Small unilamellar liposomes of $0.7 \mathrm{mM}$ DMPC were prepared on the basis of the injection method of Kremer et al. (15). Typically, $0.380 \mathrm{ml}$ of an ethanolic solution, which was $9.21 \mathrm{mM}$ in DMPC and $66 \mu \mathrm{M}$ in $\mathrm{ZnPC}$ or AlPHCl, was injected with a syringe into $5 \mathrm{ml} \mathrm{PBS,} \mathrm{pH} \mathrm{7.4.} \mathrm{The} \mathrm{injection}$ was performed at $46^{\circ} \mathrm{C}$ under magnetic stirring and at a rate of $1 \mu \mathrm{l} / \mathrm{s}$. Mixed liposomes were prepared by adding ethanolic solutions of cholesterol (50\% DMPC, w/w) or cardiolipin $(30 \% \mathrm{DMPC}, \mathrm{w} / \mathrm{w})$ to the ethanolic solution.

\section{Steady-state spectroscopic measurements}

Absorbance spectra were recorded with a Hitachi U-3000 spectrophotometer and fluorescence spectra were recorded with a Hitachi F-4500 spectrofluorometer. ZnPC and $\mathrm{AlPHCl}$ solutions were excited at $600 \mathrm{~nm}$ wavelength and their fluorescence emission was recorded in the $600-800 \mathrm{~nm}$ range. All measurements were made at $25 \pm 2^{\circ} \mathrm{C}$. Bandwidths were fixed at $5 \mathrm{~nm}$ for excitation and emission.

\section{Determination of the dimerization equilibrium constants}

In order to investigate the aggregation state of a phthalocyanine solution and to determine the equilibrium dimerization constant $\left(K_{\mathrm{D}}\right)$, the absorbance spectra of a series of phthalocyanine solutions were monitored with increasing phthalocyanine concentrations. $K_{\mathrm{D}}$ for $\mathrm{ZnPC}$ and $\mathrm{AlPHCl}$ were studied in the following medium: a) organic solution (DMSO for $\mathrm{AlPHCl}$ and pyridine for $\mathrm{ZnPC}$ ), and b) DMPC liposomal medium for both. 
The $K_{\mathrm{D}}$ for the equilibrium $2 \mathrm{M} \leftrightarrow \mathrm{D}$ is defined as

$K_{\mathrm{D}}=[\mathrm{D}] /[\mathrm{M}]^{2}$

where $[\mathrm{M}]$ and $[\mathrm{D}]$ are the molar concentrations of the monomer and the dimer, respectively. The total concentration of the drug being equal to

$\mathrm{C}=[\mathrm{M}]+2[\mathrm{D}]$

straightforward calculations lead to

$[\mathrm{M}]=\frac{\left[-1+\sqrt{\left(1+8 K_{\mathrm{D}} \mathrm{C}\right)}\right]}{4 K_{\mathrm{D}}}$

$[\mathrm{D}]=\frac{\left[1+4 K_{\mathrm{D}} \mathrm{C}-\sqrt{\left(1+8 K_{\mathrm{D}} \mathrm{C}\right)}\right]}{8 K_{\mathrm{D}}}$

The absorbance of a solution containing both the monomer and the dimer is given by

Abs $=\left(\varepsilon_{\mathrm{M}}[\mathrm{M}]+\varepsilon_{\mathrm{D}}[\mathrm{D}]\right) l$

where $\varepsilon_{M}$ and $\varepsilon_{D}$ are the extinction coefficients of the monomer and the dimer, respectively, and $l$ the optical path length.

Rearrangement of the above equations leads to

$\mathrm{Abs}=\frac{\left[1-\sqrt{\left(1+8 K_{\mathrm{D}} \mathrm{C}\right)}\left[\varepsilon_{\mathrm{D}} / 2-\varepsilon_{\mathrm{M}}\right]+2 \varepsilon_{\mathrm{D}} K_{\mathrm{D}} \mathrm{C}\right]}{4 / K_{\mathrm{D}}}$

where $\mathrm{C}$ is the total concentration of the photosensitizer (monomer and dimer), $\varepsilon_{\mathrm{M}}$ and $\varepsilon_{\mathrm{D}}$ are the extinction coefficients of the monomer and dimer, respectively, and $l$ is the optical path length. $K_{\mathrm{D}}$ was determined by means of Equation 6 (16).

The plot of absorbance as a function of total drug concentration (C) according to Equation 6 was used to evaluate the proposed scheme. The values of $K_{\mathrm{D}}, \varepsilon_{\mathrm{M}}$ and $\varepsilon_{\mathrm{D}}$ were computed by nonlinear regression based on the Lavenberg-Marquardt algorithm (16) using the Igor software. An estimated value of $\varepsilon_{\mathrm{M}}$ was obtained from measurements in organic solutions within the concentration range in which the Beer-Lambert law holds for each drug. The value of $\varepsilon_{\mathrm{M}}$, determined independently, was fixed in Equation 6 and the absorbance data were fitted (allowed to vary) to obtain $\varepsilon_{\mathrm{D}}$ and $K_{\mathrm{D}}$ by a nonlinear least square procedure (16).

\section{Fluorescence quenching studies}

The quenching of the fluorescence emission by $\mathrm{ZnPC}$ and $\mathrm{AlPHCl}$ incorporated into unilamellar liposomes of DMPC in the presence and absence of additives (cholesterol or cardiolipin) was studied using $\mathrm{MV}^{2+}$, $\mathrm{AQDS}^{2-}$ and $\mathrm{AQS}^{-}$as quenchers. Phthalocyanine solutions $(5.0 \mu \mathrm{M})$ were excited at $600 \mathrm{~nm}$ and their fluorescence emission was recorded in the range of $640-750 \mathrm{~nm}$. The fluorescence quenching data obtained were analyzed by Stern-Volmer formalism (17): $\mathrm{F}_{0} / \mathrm{F}=1+K_{\mathrm{SV}}$ $[Q]$, which relates the decrease in fluorescence intensity $\left(\mathrm{F}_{0} / \mathrm{F}\right)$ to quencher concentration $[\mathrm{Q}]$; $K_{\mathrm{SV}}$ is the Stern-Volmer quenching constant.

\section{Interaction of $\mathrm{ZnPC}$ and $\mathrm{AIPHCl}$ with bovine serum albumin in organic and liposomal medium}

The interaction of the phthalocyanines $\mathrm{ZnPC}$ and AlPHCl with BSA was studied spectrofluorometrically at $25^{\circ} \mathrm{C}$ by the "double logarithmic plot" (18). For each drug tested, two sets of experiments were performed. In the first, solutions of phthalocyanines at low concentration in PBS were titrated with increasing BSA concentrations in order to determine maximum quenching, $\mathrm{F}_{\infty}$. The relative fluorescence intensities of BSA saturated with phthalocyanines, $\mathrm{F}_{\infty}$, were extrapolated from the experimental data by plotting $1 /\left(\mathrm{F}_{0}-\mathrm{F}\right)$ against $1 /[\mathrm{P}]$, where $\mathrm{F}$ is the measured fluorescence of a solution containing the protein and the phthalocyanine, $\mathrm{F}_{0}$ is the fluorescence of a solution of protein alone, and $[\mathrm{P}]$ is the phthalocyanine concentration. In the second, a protein system held at constant concentration (absorbance at an excitation wavelength of no more than 0.05 ) was titrated with small aliquots of concentrated phthalocyanine solutions. In both ex- 
periments, the total dilution was kept below $2 \%$. The fluorescence of BSA was excited at $280 \mathrm{~nm}$ and recorded between 300 and 450 $\mathrm{nm}$, with excitation and emission bandpasses of $5 \mathrm{~nm}$. Fluorescence data were treated according to the methods of Lehrer and Fashman (19) and Chipman et al. (20), with $\log \left[\left(\mathrm{F}_{0}-\mathrm{F}\right) /\left(\mathrm{F}-\mathrm{F}_{\infty}\right)\right]$ being plotted against $\log [\mathrm{P}]$. The slope of the plot obtained gives $\mathrm{N}$, the number of binding sites, and the value of $\log [\mathrm{P}]$ at $\log \left[\left(\mathrm{F}_{0}-\mathrm{F}\right) /\left(\mathrm{F}-\mathrm{F}_{\infty}\right)\right]=0$ is equal to the logarithm of the dissociation constant $K_{\text {diss. }}$. The reciprocal of $K_{\text {diss }}$ is the binding constant $K_{\mathrm{b}}$.

\section{Triplet state measurements}

All measurements were made using ZnPC and $\mathrm{AlPHCl}(5.0 \mu \mathrm{M})$ in organic medium (pyridine and DMSO, respectively), and liposomal medium in the presence and absence of the additives cholesterol and cardiolipin. Triplet state was investigated using a laser flash photolysis apparatus which allows the simultaneous capture of the transient absorbance spectrum and of the transient kinetics at a single wavelength. The system uses an Nd-YAG laser as the excitation source (SURELITE I-10 of Continuum, Santa Barbara, CA, USA), operating at 355 $\mathrm{nm}$, ca. $50 \mathrm{~mJ} /$ pulse to give 10 -ns pulses. The decay kinetics of the triplet state of $\mathrm{ZnPC}$ and $\mathrm{AlPHCl}$ was recorded at 480 and $490 \mathrm{~nm}$, respectively - the triplet state absorbance maximum. Ten laser shots were averaged for each measurement. Decay profiles were fitted by an interactive nonlinear least-squares routine method using a data analysis software package from Edinburgh Instruments (Edinburgh, UK) on a personal computer.

\section{Results and Discussion}

\section{Determination of the dimerization equilibrium constants}

Like most phthalocyanines, $\mathrm{ZnPC}$ and
$\mathrm{AlPHCl}$ are prone to aggregation. In aqueous solution, even the soluble phthalocyanines (tetrasulfonated) display a typically aggregated spectrum even at low concentrations. Previous studies with another soluble photosensitizer (bacteriochlorin a) in PBS revealed that this hydrophilic photosensitizer is strongly aggregated in its dimeric form with a $K_{\mathrm{D}}$ estimated to be $10^{6} \mathrm{M}^{-1}(21)$. In the present study, it was not possible to determine the $K_{\mathrm{D}}$ of the drugs $\mathrm{ZnPC}$ and $\mathrm{AlPHCl}$ in aqueous solutions due to the lack of solubility in this medium. The solubilization of the drugs within DMPC liposomes induced dye monomerization and permitted us to evaluate the $K_{\mathrm{D}}$ in DMPC medium.

The trend of the phthalocyanines $\mathrm{ZnPC}$ and $\mathrm{AlPHCl}$ to form aggregates and the equilibrium $K_{\mathrm{D}}$ were analyzed and evaluated using absorbance spectroscopic analysis of the Q band at $673 \mathrm{~nm}$. It was observed that, at least up to the concentration of $5.0 \mu \mathrm{M}$, the increase of absorbance with phthalocyanine concentration was linear in organic and liposomal medium (Figure 2). This behavior implies that, at least within this concentration range ( 0 to ca. $5.0 \mu \mathrm{M}$ ), these phthalocyanines are in the monomeric state, in agreement with the Beer-Lambert law. In contrast, the behavior observed in liposomal medium

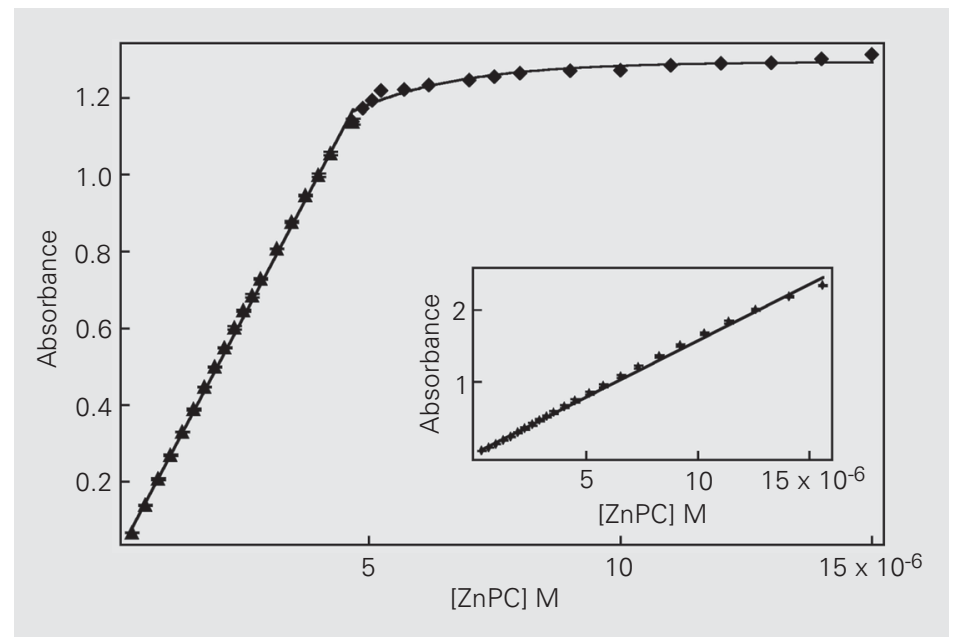

Figure 2. Variation in the absorbance $(673 \mathrm{~nm})$ as a function of zinc phthalocyanine ( $\mathrm{ZnPC}$ ) concentration in DMPC liposomes and in organic solution (pyridine) in the inset. 
in the concentration range above $5.0 \mu \mathrm{M}(5$ to $15 \mu \mathrm{M}$ ) indicated that at higher concentrations a progressive change in the system occurred, indicating a typical aggregation process of the drugs. An example of the procedure for processing the data obtained is illustrated in Figure 2 for $\mathrm{ZnPC}$. The points show the experimental results and were adjusted by nonlinear regression in the full concentration range studied ( 0 to $15.0 \mu \mathrm{M}$ ).

The $K_{\mathrm{D}}$ of $\mathrm{ZnPC}$ and $\mathrm{AlPHCl}$ in organic and liposomal medium obtained by nonlinear regression in the full concentration range studied ( 0 to $15.0 \mu \mathrm{M}$ ) are shown in Table 1 .

$K_{\mathrm{D}}$ for photosensitizers such as porphyrins and phthalocyanines range from $10^{4}$ to $10^{7} \mathrm{M}^{-1}$ and depend on the solvent and temperature (22). The results obtained confirmed that $K_{\mathrm{D}}$ strongly depend on the medium, since in liposome medium aggregation tends to be higher than in organic medium. In addition, the results indicate that the complexed metal ions zinc and aluminum do not interfere with the tendency to aggregate (same stability - same order of magnitude of $K_{\mathrm{D}}$ values for both drugs, i.e., $\mathrm{ZnPC}$ and $\mathrm{AlPHCl}$, in liposomal medium). We observed that the incorporation of the drugs into liposomes increased the $K_{\mathrm{D}}$ by one order of magnitude. These results are in agreement with the behavior expected for phthalocyanines incorporated into liposomes. However, the increase of one order of magnitude in the $K_{\mathrm{D}}$ of

Table 1. Dimerization constants of $\mathrm{ZnPC}$ and $\mathrm{AlPHCl}$ at $25^{\circ} \mathrm{C}$ in organic solution and DMPC liposomes.

\begin{tabular}{lcc} 
Medium & \multicolumn{2}{c}{$K_{\mathrm{D}}\left(\mathrm{M}^{-1}\right)$} \\
\cline { 2 - 3 } & ZnPC & AlPHCl \\
\hline Organic & $3.6 \times 10^{4}$ & $3.7 \times 10^{4}$ \\
DMPC & $1.0 \times 10^{5}$ & $1.5 \times 10^{5}$
\end{tabular}

AlPHCl = chloroaluminum phthalocyanine; DMPC $=$ dimyristoyl phosphatidylcholine; $\mathrm{DMSO}=$ dimethyl sulfoxide; $K_{D}=$ dimerization constant; organic medium = pyridine for $\mathrm{ZnPC}$ and DMSO for AlPHCl; $\mathrm{ZnPC}=$ zinc phthalocyanine these phthalocyanines after their incorporation into liposomes is not a negative factor. A $K_{\mathrm{D}}$ with an order of magnitude of $10^{5}$ is not a high value for these types of drugs (18). The advantage of the incorporation of these hydrophobic drugs into drug delivery systems such as liposomes is the increase in their solubility, permitting their systemic administration.

\section{Fluorescence quenching studies}

The locations of $\mathrm{ZnPC}$ and $\mathrm{AlPHCl}$ in DMPC liposomes mixed with cholesterol $(50 \%$ DMPC, $w / w)$ or cardiolipin $(30 \%$ DMPC, w/w) were evaluated and compared with those obtained for the drugs incorporated into DMPC liposomes in the absence of these additives to assess how the distribution of the phthalocyanines and the interaction mode of the quenchers with them are affected by the physicochemical properties of the liposomes in the presence of the additives.

The quenching of $\mathrm{ZnPC}$ and $\mathrm{AlPHCl}$ by the quenchers was monitored by measuring the decrease in the emission intensity with excitation at $600 \mathrm{~nm}$ as a function of quencher concentration. Stern-Volmer plots were constructed from the relative integrated fluorescence intensity $(640-750 \mathrm{~nm})$. When ZnPC and $\mathrm{AlPHCl}$ were incorporated into DMPC liposomes in the presence and absence of additives at $25^{\circ} \mathrm{C} \mathrm{MV}^{2+}, \mathrm{AQS}^{-}$, and $\mathrm{AQDS}^{2-}$ yielded linear fluorescence quenching plots. Table 2 shows the quenching parameters obtained in these studies.

The quenchers used in the present investigation did not penetrate the lipid bilayer and the quenching occurred by an electron transfer process (14). According to Ford and Tollin (14), in liposomal systems $\mathrm{MV}^{2+}$ is oriented co-planar to the liposomal surface in order to maximize the contact between the polar and nonpolar parts of the quencher and the liposome. On the other hand, $\mathrm{AQS}^{-}$interacts perpendicularly to the bilayer surface, 
with the sulfonate group probably oriented in an opposite direction to the liposome. Thus, in liposomal systems $\mathrm{MV}^{2+}$ interacts only with the drugs located in the outer part of the lipid bilayer, while $\mathrm{AQS}^{-}$and $\mathrm{AQDS}^{2-}$ interact with the drugs in both the inner and outer regions of the lipid bilayer. However, neither of these quenchers is able to diffuse into the lipid bilayer (quenching by an electron transfer process). Thus, the $K_{\mathrm{SV}}$ values obtained for the quenching by $\mathrm{MV}^{2+}$ belong to the drug population more exposed to the external medium, while $K_{\mathrm{Sv}}$ values obtained for anthraquinone-type quenchers represent the weighted average of the $K_{\mathrm{Sv}}$ 's of both classes of fluorophores (inner and outer).

The lower $K_{\mathrm{SV}}$ values for $\mathrm{MV}^{2+}$ compared with those for the anthraquinone quenchers (Table 2) in DMPC liposomes indicate that the drugs are incorporated into the bilayer structure, in the inner part of the liposomal structure, which lowers the accessibility of $\mathrm{MV}^{2+}$ to these drugs. The results showed that $\mathrm{AQS}^{-}$and $\mathrm{AQDS}^{2-}$ interacted with $\mathrm{ZnPC}$ and $\mathrm{AlPHCl}$ in both the inner and outer lipid bilayers by an electron transfer process, although the efficiency of fluorescence quenching of the phthalocyanines by $\mathrm{AQDS}^{2-}$ was lower compared with $\mathrm{AQS}^{-}$, as suggested by the drop of the $K_{\mathrm{SV}}$ values obtained for AQDS ${ }^{2-}$ (Table 2). In this case, the $K_{\mathrm{SV}}$ values of $\mathrm{AQDS}^{2-}$ are related to the molecules of $\mathrm{ZnPC}$ and AlPHCl mainly located in the outermost regions of the lipid bilayer, and the efficiency of quenching was higher for AlPHCl than for $\mathrm{ZnPC}$.

Different locations of the phthalocyanines in the lipid bilayer can be deduced from the changes in these $K_{\mathrm{Sv}}$ values under the same experimental conditions. The $\mathrm{AQS}^{-}$quencher was more efficient for both probes. However, AQDS ${ }^{2-}$ showed a higher quenching efficiency for AlPHCl than for ZnPC. These results indicate that $\mathrm{AlPHCl}$ was located closer to the bilayer/water interface than $\mathrm{ZnPC}$. The $K_{\mathrm{SV}}$ values for these compounds are in agreement with the values found for other photosensitizers in other drug delivery systems (23).

In the liposomes containing additives, the fluorescence quenching of the phthalocyanines by $\mathrm{MV}^{2+}$ was negligible. The efficiency of quenching by AQDS ${ }^{2-}$ in liposomal medium with additives was also lower than the efficiency of quenching with $\mathrm{AQS}^{-}$ (Table 2). The presence of additives decreased the $K_{\mathrm{SV}}$ for both drugs studied.

Clusters of cholesterol appear in vesicles at mol fractions above 0.67 . Mol fractions higher than 0.5 of cholesterol are required for cluster formation with phosphatidylcholine (24-26). When the cholesterol content exceeds the saturation limit in phospholipid membranes, crystalline cholesterol monohydrate forms $(27,28)$. In general, for choline phospholipids the saturation limit is $\approx 50 \%$ cholesterol, although a higher solubility $(65 \%)$ has been reported by Huang et al. (29) and a solubility higher than $60 \%$ was obtained by Guo and Hamilton (27). In this way, the cholesterol concentration used in the experiments $(50 \%$ of total lipid, w/w) does not exceed the saturation limit in phosphatidylcholine membranes and does not form crystalline cholesterol monohydrate.

In a membrane bilayer, cholesterol inserts normal to the plane of the bilayer, with its hydroxyl group in close vicinity to the phospholipid polar heads and its alkyl side chain extending towards the bilayer center.

Table 2. Fluorescence quenching parameters of $\mathrm{ZnPC}$ and $\mathrm{AIPHCl}(5.0 \mu \mathrm{M})$ in DMPC liposomes in the presence and absence of additives at $25^{\circ} \mathrm{C}$

\begin{tabular}{|c|c|c|c|c|c|c|}
\hline & \multicolumn{6}{|c|}{$K_{\mathrm{SV}}\left(\mathrm{M}^{-1}\right)$} \\
\hline & \multicolumn{3}{|c|}{$5 \mu \mathrm{M} \mathrm{ZnPC}$} & \multicolumn{3}{|c|}{$5 \mu \mathrm{M} \mathrm{AlPHCl}$} \\
\hline & $\mathrm{MV}^{2+}$ & $\mathrm{AOS}^{-}$ & AODS $2-$ & $\mathrm{MV}^{2+}$ & $\mathrm{AOS}^{-}$ & $\mathrm{AODS}^{2-}$ \\
\hline Without addition & - & 269.8 & 195 & 2.0 & 239.0 & 283 \\
\hline $50 \%$ cholesterol & - & 131.9 & 121 & - & 93.0 & 172 \\
\hline $30 \%$ cardiolipin & - & 45.1 & 59 & - & 38.1 & 56 \\
\hline
\end{tabular}

AlPHCl = chloroaluminum phthalocyanine; $\mathrm{AQDS}^{2-}=$ 9,10-anthraquinone-2,6disulfonate; $\mathrm{AOS}^{-}=9,10$-anthraquinone-2-sulfonate; $K_{\mathrm{SV}}=$ Stern-Volmer quenching constant; $\mathrm{MV}^{2+}=$ methylviologen; $\mathrm{ZnPC}=$ zinc phthalocyanine. 
Cholesterol is intercalated in the membrane parallel to the phospholipid hydrocarbon chains, and the phospholipid carbons at positions 2-10 have been estimated to lie in close proximity to the sterol tetracyclic ring structure $(30,31)$. Although cholesterol can enhance lateral separation of lipids in bilayers consisting of a single lipid species, resulting in a higher water permeability in phosphatidylcholine bilayers and creating a cavity that was probably occupied by water molecules (32-34), the phthalocyanines did not become more accessible to the quenchers located in the external medium, as can be seen in Table 2 (lower $K_{\mathrm{SV}}$ values in the presence of cholesterol). Thus, the incorporation of cholesterol into liposomes caused a change in the distribution of the molecules of $\mathrm{ZnPC}$ and $\mathrm{AlPHCl}$ from the outer to the inner region of the lipid bilayer. This cholesterol-induced redistribution of $\mathrm{ZnPC}$ and AlPHCl may be correlated with a change in the distribution of cholesterol itself (32-34). Above 30\% DMPC (w/w), cholesterol preferentially dissolves in the inner lipid bilayer. When the liposomes contain equimolar amounts of phosphatidylcholine and cholesterol, the inner bilayer contains about $65 \%$ DMPC of cholesterol (w/w) and the external region of the bilayer contains about $40 \%$ DMPC (w/w) (32-34). If the drugs had been located near the bilayer/water interface, they would have become more accessible to the water-soluble quenchers (outer location) in liposomes containing cholesterol than in liposomes without it (14). Consequently, these results prove the inner location of the drugs in the lipid bilayer with cholesterol, and not available to the quenchers, since a drop in quenching efficiency was noted after the addition of the additive.

Cardiolipin is found in the inner membrane of mitochondria (35). The results with cardiolipin demonstrate that the insertion of this lipid into the bilayer shows the same behavior as observed for cholesterol. This suggests a preferential distribution of $\mathrm{ZnPC}$ and $\mathrm{AlPHCl}$ in cardiolipin-rich domains of the inner part of the lipid bilayer and its exclusion from DMPC-containing domains.

The results obtained here show that the additives cholesterol and cardiolipin promote a change of the drug molecules towards the inner part of the lipid bilayer, according to the lower $K_{\mathrm{Sv}}$ values obtained after the incorporation of the additives into the liposomes (Table 2), but $\mathrm{ZnPC}$ continues to be more internalized than $\mathrm{AlPHCl}$ (lower $K_{\mathrm{SV}}$ values obtained for $\mathrm{ZnPC}$ when compared with those obtained for AlPHCl for the quenching by AQDS ${ }^{2-}$ ) (Table 2). The displacement of the drug molecules to the inner part of the lipid bilayer is probably related to their tendency to prefer hydrophobic environments.

\section{Interaction of $\mathrm{ZnPC}$ and $\mathrm{AlPHCl}$ with bovine serum albumin in organic and liposomal medium}

The interaction of serum albumin with phthalocyanines quenches the fluorescence of this protein, providing a means to assess binding quantitatively. The interaction between $\mathrm{ZnPC}$ and $\mathrm{AlPHCl}$ with BSA can be followed by fluorescence spectroscopy, as illustrated in Figure 3. $\mathrm{ZnPC}$ and $\mathrm{AlPHCl}$ quench the fluorescence of BSA in proportion to the amount of drug added. The fluorescence intensity of BSA changes with the concentration of phthalocyanine in a way consistent with a reversible formation of a complex between BSA and the drugs.

The inset in Figure 4 provides a linear plot of $\mathrm{F}_{0} / \Delta \mathrm{F}$ versus $1 /[\mathrm{P}]$ for the binding of ZnPC-BSA, used for the determination of the fluorescence intensity of BSA saturated with the phthalocyanines $\left(\mathrm{F}_{\infty}\right)([\mathrm{P}]=$ phthalocyanine concentration). As described in the experimental section, $K_{\text {diss }}$ was calculated from the slope and interception of the plot of $\log \left[\left(\mathrm{F}_{0}-\mathrm{F}\right)\left(\mathrm{F}-\mathrm{F}_{\infty}\right)\right]$ versus $\log [\mathrm{P}]$ (an example of such double-logarithmic plot obtained for $\mathrm{ZnPC}$ is given in Figure 4). In 
addition, the slope of the plot gives the number of the sites of the protein able to bind $\mathrm{ZnPC}$ and $\mathrm{AlPHCl}$ molecules.

The association constants obtained for the phthalocyanines studied are presented in Table 3, together with the binding stoichiometry of the complex formed.

Previous studies have indicated that phthalocyanines without $\mathrm{SO}_{3}{ }^{-}$groups adjacent to iso-indole rings exhibit a high affinity binding site constant of $1-4 \times 10^{4} \mathrm{M}^{-1}(36,37)$. Other studies (38) have reported mesoporphyrin IX and magnesium mesoporphyrin partitioning between liposome and serum albumin with $K_{\mathrm{b}}$ of $2.5 \times 10^{7} \mathrm{M}^{-1}$ and $1.7 \times 10^{7} \mathrm{M}^{-1}$. Our results agree with these data (Table 3 ).

The present results show that when phthalocyanines are incorporated into DMPC liposomes, their association with BSA is increased in comparison with organic medium. Calorimetric titration studies indicated the binding of empty liposomes to the albumin species. The albumin molecules from different species adsorb strongly to phosphatidylcholine liposomes due mainly to the action of hydrophobic dehydration forces and entropy gain (39). In this way, the liposomes themselves increase the $K_{\mathrm{b}}$ to BSA. The encapsulation of the drug in the liposomes increases the already high association of the liposome/drug complex with BSA. The presence of a strong binding site and several weaker sites was observed for $\mathrm{ZnPC}$ and $\mathrm{AlPHCl}$ incorporated into liposomes. The present results indicate that the association of the drugs with lipid-based delivery systems affects their interaction with serum albumin. Liposomes of DMPC increase the binding of $\mathrm{ZnPC}$ and $\mathrm{AlPHCl}$ to $\mathrm{BSA}$, which should increase the selectivity of tumor targeting by these phthalocyanines, since BSA can deliver the bound drugs to the vascular stroma of the tumors (7).

\section{Triplet state measurements}

The triplet state properties of $\mathrm{ZnPC}$ and
AlPHCl are similar. The transient spectra of $\mathrm{ZnPC}$ and $\mathrm{AlPHCl}$ in liposomal medium (in the presence and absence of additives) resemble those obtained for a homogeneous solution (organic medium). Figure 5 illustrates the transient spectrum of $\mathrm{ZnPC}$ (5.0 $\mu \mathrm{M})$ incorporated into DMPC liposomes in the presence and absence of cholesterol and

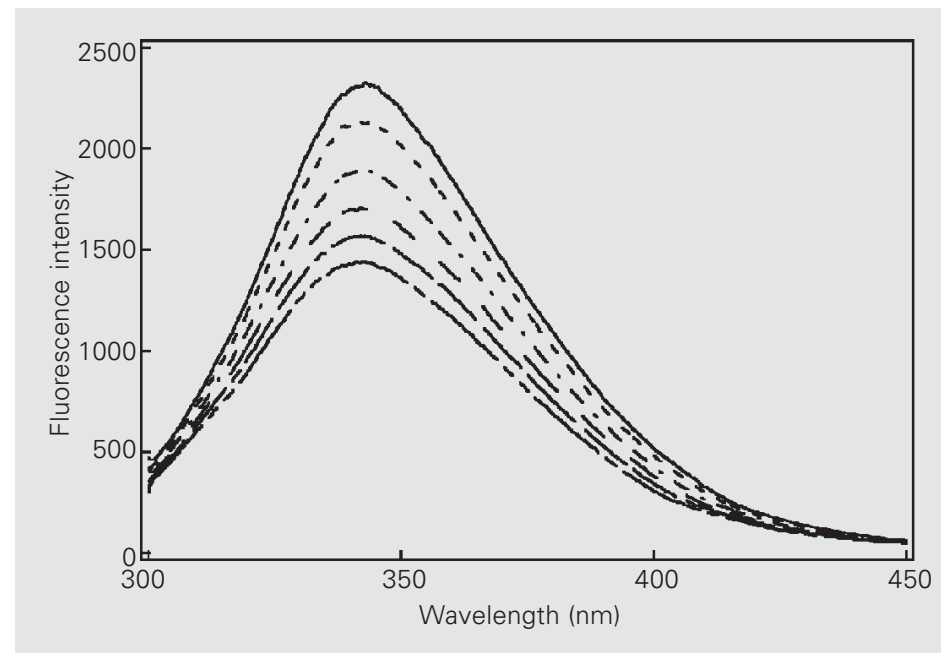

Figure 3. Effect of zinc phthalocyanine concentration on the fluorescence spectrum of bovine serum albumin. From the top, in order of decreasing fluorescence intensity with excitation at $290 \mathrm{~nm}, \mathrm{ZnPC}$ concentration was $0.00,0.13$, $0.38,0.64,0.89$, and $1.14 \mu \mathrm{M}$.

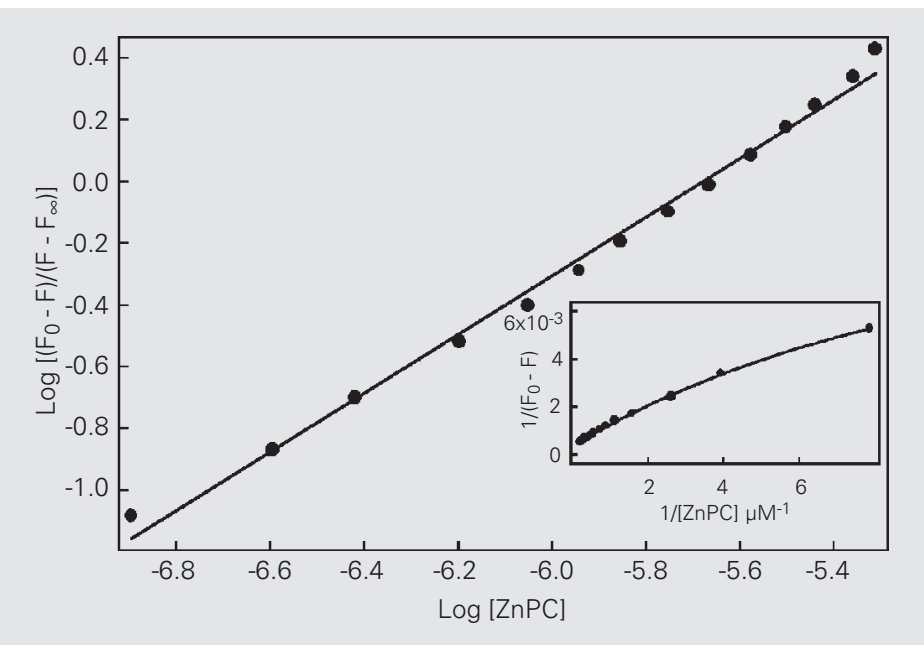

Figure 4. Double log plot of the quenching of protein fluorescence by zinc phthalocyanine in organic medium. Inset, Double reciprocal plot in organic medium ( $F=$ measured fluorescence of a solution containing the protein and the phthalocyanine, $F_{0}$ is the fluorescence of a solution of protein alone, $F_{\infty}$ is the fluorescence intensity of BSA saturated with $\mathrm{ZnPC}$, and $[\mathrm{PC}]$ is the $\mathrm{ZnPC}$ concentration). 
Table 3. Equilibrium constants of $\mathrm{ZnPC}$ and $\mathrm{AIPHCl}$ binding to BSA.

\begin{tabular}{|c|c|c|c|c|}
\hline \multirow[t]{2}{*}{ Medium } & \multicolumn{2}{|r|}{$\mathrm{ZnPC}$} & \multicolumn{2}{|c|}{$\mathrm{AlPHCl}$} \\
\hline & $\mathrm{N}$ & $K_{\mathrm{b}}\left(\mathrm{M}^{-1}\right)$ & $\mathrm{N}$ & $K_{\mathrm{b}}\left(\mathrm{M}^{-1}\right)$ \\
\hline Organic & 1.05 & $0.71 \times 10^{6}$ & 1.37 & $1.30 \times 10^{7}$ \\
\hline DMPC liposomes & 1.07 & $4.86 \times 10^{7}$ & 1.11 & $3.10 \times 10^{8}$ \\
\hline
\end{tabular}

$\mathrm{AlPHCl}=$ chloroaluminum phthalocyanine; $\mathrm{DMPC}=$ dimyristoyl phosphatidylcholine; $K_{\mathrm{b}}=$ binding constant; $\mathrm{N}=$ number of binding sites; organic medium = pyridine for $\mathrm{ZnPC}$ and DMSO for $\mathrm{AlPHCl} ; \mathrm{ZnPC}=$ zinc phthalocyanine.

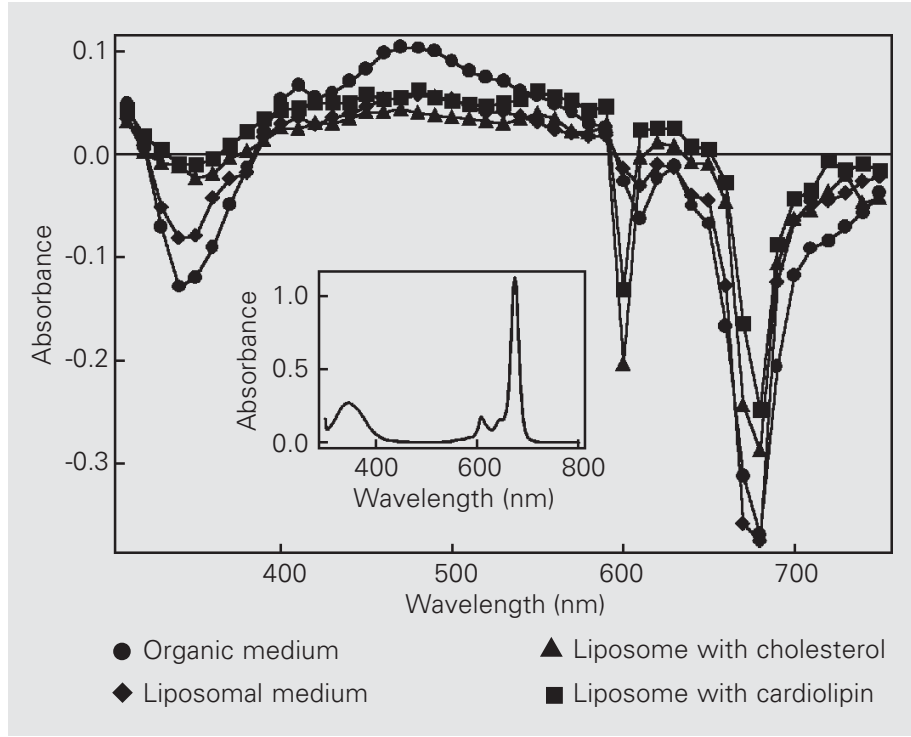

Figure 5. Transient absorption spectra of zinc phthalocyanine (ZnPC) in organic (pyridine) and liposomal medium, in the presence and absence of the additives - liposome with cholesterol, and liposome with cardiolipin - after excitation at $670 \mathrm{~nm}(50 \mathrm{~mJ}$ per pulse), showing the absorption of the triplet state centered at $\lambda=480 \mathrm{~nm}$ and the bleach of the ground state centered at around 350 and $680 \mathrm{~nm}$. Inset, Absorption spectra of ZnPC in organic medium.

Table 4. Triplet lifetimes $\left(\tau_{\top}\right)$ of $\mathrm{ZnPC}$ and AIPHCl in organic and liposomal medium in the presence of $\mathrm{O}_{2}$.

\begin{tabular}{lcc}
\hline Medium & ZnPC & $\mathrm{AlPHCl}$ \\
\hline Organic medium & 0.30 & 0.80 \\
DMPC & 0.84 & 1.28 \\
DMPC with cholesterol & 1.35 & 4.62 \\
DMPC with cardiolipin & 1.28 & 2.50
\end{tabular}

$\mathrm{AlPHCl}=$ chloroaluminum phthalocyanine; $\mathrm{DMPC}=$ dimyristoyl phosphatidylcholine; organic medium = pyridine for $\mathrm{ZnPC}$ and DMSO for $\mathrm{AlPHCl}$; $\mathrm{ZnPC}=$ zinc phthalocyanine. cardiolipin, and also serves to illustrate the triplet-triplet absorbance centered at $480 \mathrm{~nm}$, together with the Soret and Q band ground state bleaches.

Triplet lifetimes $\left(\tau_{\mathrm{T}}\right)$ were calculated from kinetic analysis of the transient decays, carried out using the fitting program of the apparatus itself and are shown in Table 4.

The triplet state reacts with forms of molecular oxygen $\left(\mathrm{O}_{2}\right)$ by an energy transfer process leading to singlet oxygen that is the key agent in cell damage in PDT. Transient absorbance spectra for the drugs obtained by laser flash photolysis showed the presence of transient species, which decayed monoexponentially with the characteristic lifetimes shown in Table 4. As a consequence of the incorporation, triplet lifetimes are increased in liposomes in comparison with homogeneous solution. The increase in the triplet lifetimes is attributed to the ability of the bulk aqueous phase to interact with the sensitizer at the binding site. Therefore, we conclude that the increase in the triplet state lifetimes of $\mathrm{ZnPC}$ and $\mathrm{AlPHCl}$ as a function of the addition of cholesterol and cardiolipin to liposomes results from a progressive reduction in the exposure of the sensitizer to the bulk aqueous phase, confirming the results obtained in the quenching studies. Under identical conditions, the maximum triplet lifetimes of $\mathrm{ZnPC}$ are shorter than those for $\mathrm{AlPHCl}$, demonstrating that $\mathrm{AlPHCl}$ is more internalized than $\mathrm{ZnPC}$.

The behavior observed in these studies implies that, at least up to ca. $5.0 \mu \mathrm{M}$, both phthalocyanines studied ( $\mathrm{ZnPC}$ and $\mathrm{AlPHCl})$ are in the monomeric state in both media (liposomal and organic), in agreement with the Beer-Lambert law. The increase of one order of magnitude in the dimerization constant of the phthalocyanines after their incorporation into liposomes does not rule out the use of this carrier, considering the increased solubility of the drugs after incorporation into the drug delivery system, allowing their systemic administration, and the increased 
binding with BSA which should increase the selectivity of tumor targeting. As a consequence of the incorporation, triplet lifetimes are increased in liposomes compared with homogeneous solution. Thus, the results obtained clearly indicate that the incorporation of these drugs into DMPC liposomes maximizes their photodynamic action, allowing an effective activity of the photosensitizerliposome complex against cancer.

The results of the present study also indicate that the distribution of $\mathrm{ZnPC}$ and $\mathrm{AlPHCl}$ in unilamellar DMPC liposomes is heterogeneous, with some predominant positions (Figure 6). In the presence of additives there is a shift of the drug molecules towards the inner part of the lipid bilayer. The results obtained indicated that $\mathrm{AlPHCl}$ and $\mathrm{ZnPC}$ are preferentially distributed in the inner part of the phospholipid bilayer of DMPC liposomes in the presence and absence of cholesterol $(50 \%$ DMPC, w/w) and cardiolipin (30\% DMPC, $\mathrm{w} / \mathrm{w})$. The increased internalization of the drugs in liposomes containing additives is a positive aspect, since in this way the drugs can be protected while they circulate in the bloodstream after systemic injection.

Silicon (IV) phthalocyanine, the first phthalocyanine approved by the FDA for clinical trials, has the highest potential of this class of drugs for PDT (40). However,

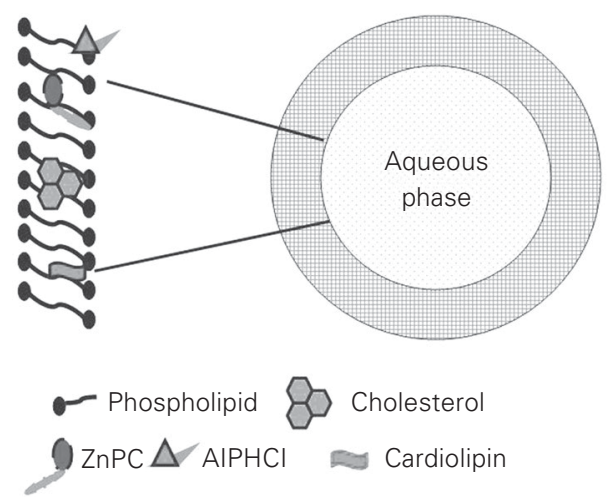

Figure 6. Preferential locations of the photosensitizers $\mathrm{ZnPC}$ and $\mathrm{AIPHCl}$ in mixed liposomes of DMPC. silicon (IV) phthalocyanine needs to be used in a Cremophor emulsion, a fact that is an obstacle to the generalized use of this drug. Cremophor has been used clinically for the delivery of hydrophobic drugs. The possibility of the use of hydrophobic phthalocyanines such as $\mathrm{ZnPC}$ and $\mathrm{AlPHCl}$ encapsulated in liposomes that act as a drug delivery system, maintaining all the basic photophysical and photochemical properties of the sensitizers, is an important result, since the liposomal vehicle is more economically viable than Cremophor. Thus, our results confirm the hypothesis that the liposomal medium represents an excellent vehicle for the administration of these drugs in biological systems, making liposomal $\mathrm{ZnPC}$ and $\mathrm{AlPHCl}$ promising candidates for PDT from the photophysical point of view.

\section{References}

1. Pandey RK (2000). Recent advances in photodynamic therapy. Journal of Porphyrins and Phthalocyanines, 4: 368-373.

2. Owens JW, Smith R, Robinson R \& Robins M (1998). Photophysical properties of porphyrins, phthalocyanines and benzochlorins. Inorganica Chimica Acta, 279: 226-231.

3. Cahn W-S, Brasseur N, La Madeleine C, Quellet R \& van Lier JE (2001). Current status of phthalocyanines in the photodynamic therapy of cancer. European Journal of Cancer, 33: 1855-1860.

4. Urizzi P, Allen CM, Langlois R, Quellet R, La Madeleine C \& van Lier JE (2001). Low density lipoprotein-bound aluminium sulphophthalocyanine: targeting tumor cells for photodynamic therapy. Journal of Porphyrins and Phthalocyanines, 5: 154-160.

5. Ruch A, Beck G, Bachor R, Akgun N, Gschwence MH \& Steiner R (1996). Dynamic fluorescence changes during photodynamic therapy in vivo and in vitro of hydrophilic Al(III) phthalocyanine tetrasul- phonated and lipophilic Zn(II) phthalocyanine administered in liposomes. Journal of Photochemistry and Photobiology. B, Biology, 36: 127-134.

6. Soncin M, Polo L, Reddi E, Jori G, Kenney ME, Cheng G \& Rodgers MAJ (1995). Effect of the delivery system on the biodistribution of $\mathrm{Ge}(\mathrm{IV})$ octabutoxy-phthalocyanines in tumour-bearing mice. Cancer Letters, 89: 101-106.

7. Storm G \& Crommelin DJA (1998). Liposomes: quo vadis. Pharmaceutical Science Technology Today, 1: 19-31.

8. Aveline BM, Hasan T \& Redmond RW (1995). The effects of aggregation, protein binding and cellular incorporation on the photophysical properties of benzoporphyrin derivative mono acid ring $A$ (BPDMA). Journal of Photochemistry and Photobiology. B, Biology, 30: 161-169.

9. Sil S, Kar M \& Chakraborti AS (1997). Studies on the interaction of 
hematoporphyrin with hemoglobin. Journal of Photochemistry and Photobiology. B, Biology, 41: 67-72.

10. Ball DJ, Wood SR, Vernon DI, Griffiths J, Dubbelman MAR \& Brown SB (1998). The characterization of three substituted zinc phthalocyanines of different charge for use in photodynamic therapy. A comparative study of their aggregation and photosensitizing ability in relation to $\mathrm{mTHPC}$ and polyhaematoporphyrin. Journal of Photochemistry and Photobiology. B, Biology, 45: 28-35.

11. Dhami S \& Phillips D (1996). Comparison of the photophysics of an aggregation and non-aggregating aluminium phthalocyanine system incorporated into unilamellar vesicles. Journal of Photochemistry and Photobiology. A, Chemistry, 100: 77-84.

12. Damoiseau X, Schuitmaker HJ, Lagerberg JW \& Hoebeke M (2001). Increase of the photosensitizing efficiency of the Bacteriochlorin a by liposome-incorporation. Journal of Photochemistry and Photobiology. B, Biology, 60: 50-60.

13. Tanielian C \& Heinrich G (1995). Effect of aggregation on the hematoporphyrin-sensitized production of singlet molecular oxygen. Photochemistry and Photobiology, 61: 131-135.

14. Ford WE \& Tollin G (1984). Chlorophyll photosensitized electron transfer in phospholipid bilayer vesicle systems: effects of cholesterol on radical yields and kinetic parameters. Photochemistry and Photobiology, 40: 249-259.

15. Kremer JMH, Esker MWJ, Pathmamanoharan C \& Wiersema PH (1977). Vesicles of variable diameter prepared by a modified injection method. Biochemistry, 16: 3932-3936.

16. Oulmi D, Maillard P, Vever-Bizet C, Momenteau M \& Brault D (1998). Glycosylated porphyrins: characterization of association in aqueous solutions by absorption and fluorescence spectroscopies and determination of singlet oxygen yield in organic media. Photochemistry and Photobiology, 67: 511-518.

17. Lakiwicz JR (1999). Principles of Fluorescence Spectroscopy. 2nd edn. Kluwer Academic Publishing/Plenum, New York.

18. Bárdos-Nagy I, Galántai R, Kaposi AD \& Fidy J (1998). Difference in the transport of metal and free-base porphyrins. Steady-state and time-resolved fluorescence studies. International Journal of Pharmacology, 175: 255-267.

19. Lehrer S \& Fashman GD (1966). The fluorescence of lysozyme and lysozyme substrate complexes. Biochemical and Biophysical Research Communications, 23: 133-138.

20. Chipman DM, Grisaro V \& Shanon N (1967). The binding of oligosaccharides containing $\mathrm{N}$-acetylglucosamine and $\mathrm{N}$-acetylmaramic acid to lysozyme. Journal of Biological Chemistry, 242: 4388-4394.

21. Damoiseau X, Tfibel F, Hoebeke M \& Fontaine-Aupart MP (2002). Effect of aggregation on bacteriochlorin a triplet-state formation: a laser flash photolysis study. Photochemistry and Photobiology, 76 : 480-485.

22. Monahan AR, Brado JA \& DeLuca AF (1972). The association of copper (II), vanadyl and zinc (II) 4,4', 4',-tetraalkylphthalocyanine dyes in benzene. Journal of Physical Chemistry, 76: 1994-1996.

23. Ricchelli F, Jori G, Gobbo S \& Tronchin M (1991). Liposomes as models to study the distribution of porphyrins in cell membranes. Biochimica et Biophysica Acta, 1065: 42-48.

24. Bittman R, Kasireddy CR, Mattjus P \& Slotte JP (1994). Interaction of cholesterol with sphingomyelin in monolayers and vesicles. Biochemistry, 33: 11776-11781.

25. Slotte JP (1992). Enzyme-catalysed oxidation of cholesterol in mixed phospholipid monolayers reveals the stoichiometry at which free cholesterol clusters disappear. Biochemistry, 31: 5472-5477.
26. Epand RM (2003). Cholesterol in bilayers of sphingomyelin or dihydrosphingomyelin at concentrations found in ocular lens membranes. Biophysical Journal, 84: 3102-3110.

27. Guo W \& Hamilton JA (1995). A multinuclear solid-state NMR study of phospholipid-cholesterol interaction. Dipalmitoylphosphatidylcholine-cholesterol binary system. Biochemistry, 34: 14174-14184.

28. Guo W, Kurze V, Huber T, Afdhal NH, Beyer K \& Hamilton JA (2002). A solid state NMR study of phospholipid-cholesterol interactions: sphingomyelin-cholesterol binary systems. Biophysical Journal, 83: 1465-1478.

29. Huang J, Buboltz JT \& Feigenson GW (1999). Maximum solubility of cholesterol in phosphatidylcholine and phosphatidylethanolamine bilayers. Biochimica et Biophysica Acta, 1417: 89-100.

30. Morrow MR, Singh D, Lu D \& Grant CW (1995). Glycosphingolipid fatty acid arrangement in phospholipid bilayers: cholesterol effects. Biophysical Journal, 68: 179-186.

31. O'Leary TJ (1993). Vibrational spectroscopy of cholesterol-lipid interactions. In: Finegold L (Editor), Cholesterol in Membrane Models. CRC Press, Boca Raton, FL, USA, 175-196.

32. Bittman R (1997). Has nature designed the cholesterol side chain for optimal interaction with cholesterol? In: Bittman R (Editor), Subcellular Biochemistry Series. Vol. 28: Cholesterol: Its Functions and Metabolism in Biology and Medicine. Plenum Press, New York, 145-172.

33. Davis JH (1993). The molecular dynamics, orientational order and thermodynamic phase equilibria of cholesterol/phosphatidylcholine mixtures: $2 \mathrm{H}$ nuclear magnetic resonance. In: Finegold L (Editor), Cholesterol in Membrane Models. CRC Press, Boca Raton, FL, USA, 67-136.

34. Ohvo-Rekilã H, Ramstedt B, Leppimäki P \& Slotte JP (2002). Cholesterol interactions with phospholipids in membranes. Progress in Lipid Research, 41: 66-97.

35. Jacobson J, Duchen MR \& Heales SJR (2002). Intracellular distribution of the fluorescent dye nonyl acridine orange responds to the mitochondrial membrane potential: implications for assays of cardiolipin and mitochondrial mass. Journal of Neurochemistry, 82: 224-233.

36. Gantchev TG, Oullet R \& van Lier JE (1999). Binding interactions and conformational changes induced by sulfonated aluminum phthalocyanines in human serum albumin. Archives of Biochemistry and Biophysics, 366: 21-30.

37. Filyasova AI, Kudelina IA \& Feafanov AV (2001). A spectroscopic study of the interaction of tetrasulfonated aluminum phthalocyanine with human serum albumin. Journal of Molecular Structure, 565-566: 173-176.

38. Bárdos-Nagy I, Galántai R \& Fidy J (2001). Effect of trehalose in low concentration on the binding and transport of porphyrins in liposome-human serum albumin system. Biochimica et Biophysica Acta, 1512: 125-134.

39. Dimitrova MN, Matsumura H, Dimitrova A \& Neitchev V (2000). Interaction of albumins from different species with phospholipid liposomes. Multiple binding sites system. International Journal of Biological Macromolecules, 27: 187-194.

40. Wohrle D, Muller S, Shopova M, Mantareva V, Spassova GVF, Ricchelli F \& Jori G (1999). Effects of delivery system on the pharmacokinetic and phototerapeutic properties of bis(methyloxyethyleneoxy) silicon-phthalocyanine in tumour-bearing mice. Journal of Photochemistry and Photobiology. B, Biology, 50: 124-128. 